\title{
Current-Voltage Characteristics of the Plasma Focus: A Deeper Look ${ }^{\dagger}$
}

\author{
Sor Heoh SAW ${ }^{1,2, *}$ and Sing $\operatorname{LEE}^{1,2,3,4}$ \\ ${ }^{1}$ Nilai University, Negeri Sembilan Malaysia, Malaysia \\ ${ }^{2}$ Institute Plasma Focus Studies, Melbourne, Australia \\ ${ }^{3}$ Centre for Plasma Research, INTI International University, Malaysia \\ ${ }^{4}$ Plasma Research Laboratory, Department of Physics, University of Malaya, Kuala Lumpur, Malaysia
}

('Corresponding author's e-mail: saw.ipfs@gmail.com)

Received: 15 December 2017, Revised: 16 November 2018, Accepted: 21 November 2018

\begin{abstract}
A capacitor bank discharges a current which is a sinusoidal function, lightly damped by unavoidable circuit resistance. When powering a plasma focus, the current waveform is further damped by the axial motion typically during the rising part of the current. The radial phase, with severe rate of change of inductance due to a rapidly collapsing current sheet to a small radius, is so severely damped over a short period near the current peak that the waveform goes into the sharp dip. This produces the well-known signature current dip of the properly-operated plasma focus. Corresponding to the inductively-caused current dip is a sharp voltage spike which typically rises to a peak value in excess of the voltage to which the capacitor is charged. These features are adequately described by circuit equations coupled to appropriate equations of motion. The loading effect of different gases due to differences in mass, differences in compressibility and differences in radiation also produces differences in the current waveforms particularly in the current dips and voltage spikes. These differences could be subtle or dramatic, as are demonstrated in this paper.
\end{abstract}

Keywords: Dense plasma focus, current-voltage characteristics, high density plasma, compressibility, radiation collapse

\section{Discharge into a short circuit}

A capacitor bank with bank parameters $L_{0}, C_{0}, R_{0}$ charged to $V_{0}$ discharges with damped sinusoidal characteristics with periodic time $\mathrm{T}$ and current amplitude $\mathrm{I}_{0}$ given as follows.

$$
\begin{gathered}
T=2 \pi\left(L_{0} C_{0}\right)^{0.5} \\
I_{0}=V_{0} /\left(L_{0} / C_{0}\right)^{0.5}
\end{gathered}
$$

An example of a capacitor bank discharge at MA level is shown in Figure 1.

\footnotetext{
${ }^{\dagger}$ Presented at the $10^{\text {th }}$ International Conference on Plasma Science and Applications 2017: October $10^{\text {th }}-11^{\text {th }}, 2017$
} 
http://wjst.wu.ac.th

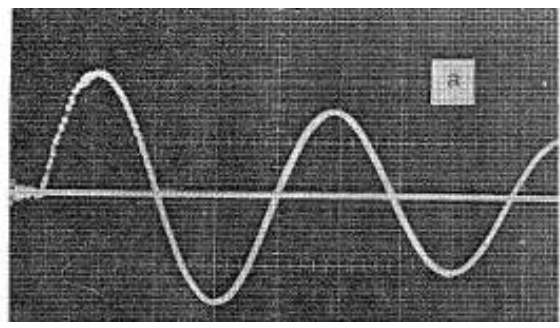

Figure 1 MA discharge current waveform of the UM bank short-circuited at $20 \mathrm{kV}$, current at first peak is $0.96 \mathrm{MA}$. The periodic time was measured at $7.6 \mu$ s [1].

\section{Capacitor bank discharged into dense plasma focus}

A capacitor discharge powers the various phases of the dense plasma focus (DPF), starting with the axial phase followed by the various radial phases. It is found that only a fraction $f_{c}$ of the capacitor discharge current $I(t)$ effectively accelerates and heats the plasma. The remnant current with fraction $\left(1-f_{c}\right)$ is left behind, likely near the insulator region of the plasma focus. The effective circuit of the DPF is shown in Figure 2, with $L_{0}$ being the static inductance and $L(t)$ being the inductance contributed by the configuration of current flowing in the plasma fronts and eventually the pinch. Figure 3 shows a comparison of discharge current for the two cases of no load (i.e. short-circuit) and DPF load [2-4].

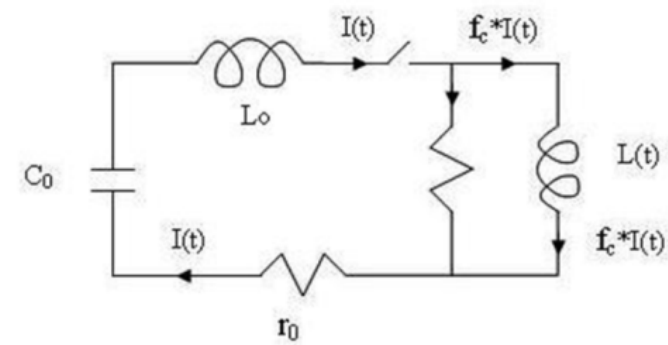

Figure 2 The DPF circuit.



Figure 3 Capacitor bank discharge currents: no load (i.e. short-circuit SC) versus DPF load [2-4]. 
http://wjst.wu.ac.th

\section{Axial phase current and voltage}

For discharge into a DPF load, the current maintains its mainly sinusoidal but distorted current, (see Figure 3). The discharge time (first half-cycle) is increased with distortion and the peak current is decreased. The effect of the axial phase dynamics is seen in comparing the short-circuited current trace and the measured (or computed) current trace from start of current to around 1.1 $\mu \mathrm{s}$. The increasingly flattened and depressed DPF current is due to the increasing inductance of the axial phase current loop, with the current sheath advancing at almost constant high axial speed towards the end of the coaxial tube. The reduced value of the peak current is due to the 'motor' back emf effect produced by the $I d L / d t$ term of the tube voltage $d(\Phi) / d t$ where $\Phi=L I f_{c}$.

The rate of change of current [2-4] is given by;

$$
\frac{d I}{d t}=\left[V_{o}-\frac{\int I d t}{C_{o}}-r_{o} I-I f_{c} \frac{\mu}{2 \pi}(\ln c) \frac{d z}{d t}\right] /\left[L_{o}+\frac{f_{c} \mu}{2 \pi}(\ln c) z\right]
$$

The tube voltage is given by;

$$
V=\frac{d}{d t}\left(L I f_{c}\right)=f_{c} I \frac{d L}{d t}+f_{c} L \frac{d I}{d t}
$$

where $L=\mu / 2 \pi(\ln c) z$ and $d L / d t$ is proportional to the axial speed $d z / d t$.

The tube voltage (see Figure 4) in the axial phase is primarily due to $f_{c} I d L / d t=f_{c}(\mu / 2 \pi)(\ln c) I d z / d t$. This primary component is moderated by the reactive $d I / d t$ term. This can be seen from the comparison of the axial phase tube voltage and the axial phase current sheet speed. It can further be understood through the concept of the motor effect. For example towards the end of the axial phase, the axial speed of the current sheet is typically at a high and almost constant speed of $10 \mathrm{~cm} / \mu \mathrm{s}$ whereas $d I / d t$ is almost zero. At that time the dominant term $f_{c} I d L / d t=f_{c}(\mu / 2 \pi)(\ln c) I d z / d t$ is approximately $5 \mathrm{kV}$ for the case of $I=350$ $\mathrm{kA}$ and typical $f_{c}=0.7$.

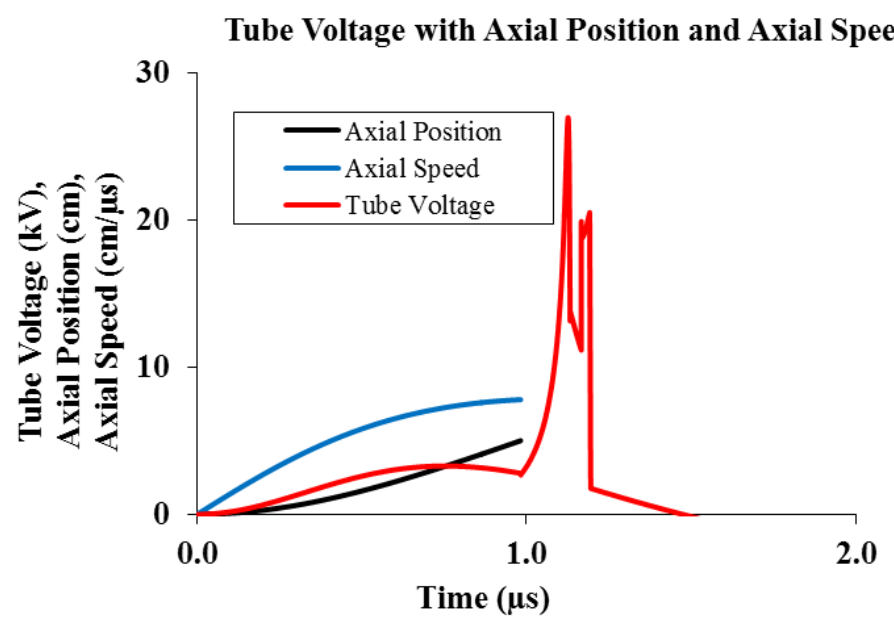

Figure 4 Comparison of axial phase tube voltage with axial speed and position. 


\section{Radial phase current and voltage}

The radial phase, with severe rate of change of inductance due to a rapidly collapsing current sheet to a small radius, is so severely damped over a short period near the current peak that the waveform goes into the observed sharp dip.

Radial phase rate of change of current [2-4] is;

$\frac{d I}{d t}=\frac{V_{o}-\frac{\int I d t}{C_{o}}-r_{o} I-f_{c} \frac{\mu}{2 \pi}\left(\ln \frac{b}{r_{p}}\right) I \frac{d z_{f}}{d t}+f_{c} \frac{\mu}{2 \pi} \frac{z}{r_{p}} I \frac{d r_{p}}{d t}}{L_{o}+f_{c} \frac{\mu}{2 \pi}(\ln c) z_{o}+f_{c} \frac{\mu}{2 \pi}\left(\ln \frac{b}{r_{p}}\right) z_{f}}$

Radial phase tube voltage [2-4] is;

$$
\begin{aligned}
& V=\frac{\mu}{2 \pi}\left[(\ln c) z_{0}+\left(\ln \frac{b}{r_{p}}\right) z_{\mathrm{f}}\right] \mathrm{f}_{\mathrm{c}} \frac{d I}{d t}+\frac{\mu}{2 \pi}\left[\left(\ln \frac{b}{r_{p}}\right) \frac{d z_{\mathrm{f}}}{\mathrm{dt}}-\frac{z_{\mathrm{f}}}{\mathrm{r}_{\mathrm{p}}} \frac{d r_{p}}{d t}\right] \mathrm{f}_{\mathrm{c}} I \\
& V=V_{1}+V_{2} \\
& V=V_{1}+V_{21} V_{22}
\end{aligned}
$$

The tube voltage (see Figure 5) at the time of the radial phase is dependent primarily on the second term $V_{2}$ in the tube voltage equation. This dependence is on the product of the current $I$ and the current sheet speed; with one component $V_{2 l}$ dependent on the elongation speed $d z_{f} / d t$ and the other component $V_{22}$ dependent on the radial speed $d r_{p} / d t$ multiplied by the factor $z_{f} / r_{p}$. The first term $V_{l}$ is the reactive term that opposes the primary causative term $V_{2}$. Table 1 shows the magnitudes of these terms for the small DPF device NX2 and the large DPF device PF1000 (highly damped device) and the hypothetical PF1000 (lightly damped (RESF=0.1) as in a typical DPF).
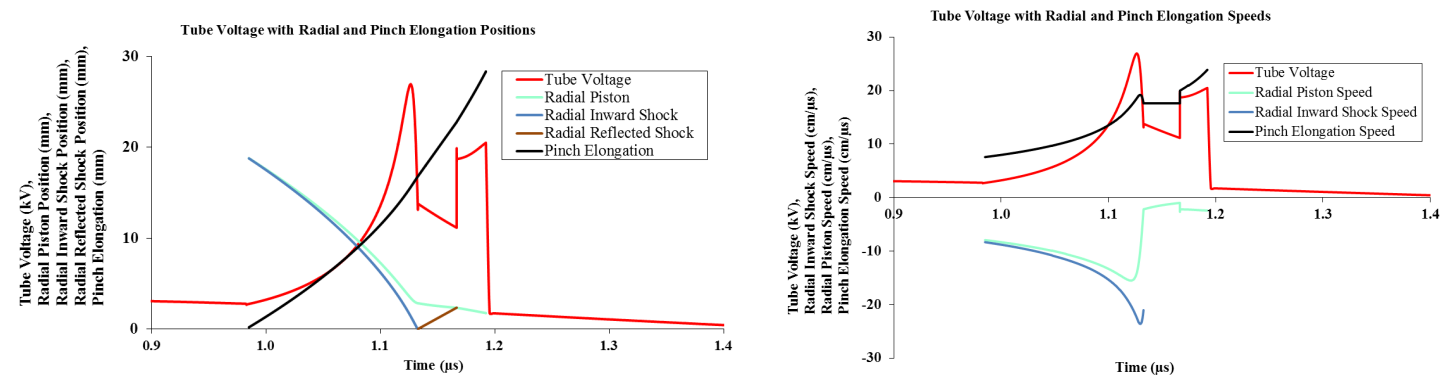

Figure 5 Comparison of radial phase tube voltage with radial phase positions and speeds. 
http://wjst.wu.ac.th

Table 1 Tube voltage and contributing terms for the small DPF NX2 and the large DPF PF1000.

\begin{tabular}{|c|c|c|c|c|c|c|}
\hline \multirow{2}{*}{$\begin{array}{c}\text { DPF } \\
\text { device }\end{array}$} & $\begin{array}{l}\text { Tube } \\
\text { voltage }(\mathrm{kV})\end{array}$ & $\begin{array}{l}d I / d t \text { *position } \\
\text { term }\end{array}$ & $\begin{array}{l}\text { speed } * I \\
\text { term }\end{array}$ & $\begin{array}{l}\left(d z_{f} / d t\right) * I \\
\text { term }\end{array}$ & $\begin{array}{l}\left(d r_{p} / d t\right)^{*} I \\
\text { term }\end{array}$ & \multirow{2}{*}{$\begin{array}{l}\text { Effective } \\
\text { beam ion energy } \\
\text { (keV) }\end{array}$} \\
\hline & $\mathbf{V}$ & $V_{1}$ & $\mathrm{~V}_{2}$ & $V_{21}$ & $V_{22}$ & \\
\hline NX2 (15 kV) & 25.1 & -10.6 & 35.7 & 17.1 & 18.6 & 75 \\
\hline PF1000 (27 kV) & 25.7 & -29.8 & 55.5 & 29.1 & 26.4 & 77 \\
\hline $\begin{array}{l}\text { PF1000 }(27 \mathrm{kV}) \\
(\mathrm{RESF}=0.1)\end{array}$ & 57.0 & -65.6 & 122.6 & 62.9 & 59.7 & 171 \\
\hline
\end{tabular}

Note that the radial speed multiplier $z_{f} / r_{p}$ becomes very large if the current sheet gets close to the axis, particularly in the case of radiatively enhanced pinches [5].

\section{Effect of thermodynamics and radiation}

The effects of thermodynamics and radiation are more easily discussed starting from the effects on the dynamics, which in turn affect the current and voltage waveforms. In a gas (plasma) heated to high temperatures, the dissociation and ionisation add to the degrees of freedom of the gas. This changes the compressibility of the plasma and affects the dynamics. This effect of the specific heat ratio (SHR) $\gamma$ on the shock speed in our code [2-4] is shown in the following shock speed equation.

$$
\frac{d r_{s}}{d t}=-\left[\frac{\mu(\gamma+1)}{\rho_{o}}\right]^{1 / 2} \frac{\mathrm{f}_{\mathrm{c}}}{\sqrt{\mathrm{f}_{\mathrm{mr}}}} \frac{I}{4 \pi r_{p}}
$$

Similar $\gamma$ interactive term affects the piston speed and column elongation. The effect of radiation is coupled into the pinch phase piston speed by the following equation.

$$
\frac{d r_{p}}{d t}=\frac{\frac{-r_{p}}{\gamma I} \frac{d I}{d t}-\frac{1}{\gamma+1} \frac{r_{p}}{z_{\mathrm{f}}} \frac{d z_{\mathrm{f}}}{\mathrm{dt}}+\frac{4 \pi(\gamma-1)}{\mu \gamma z_{\mathrm{f}}} \frac{r_{p}}{\mathrm{f}_{\mathrm{c}}^{2} I^{2}} \frac{d Q}{d t}}{\frac{\gamma-1}{\gamma}}
$$

where $d Q / d t$ is the total power gain/loss of the plasma column. In the standard code, joule heating (adding a positive component to $d Q / d t$ ), bremsstrahlung, line and recombination radiation (adding negative components to $d Q / d t$ ) are incorporated into $d Q / d t$.

By this coupling of radiation into the dynamics equation, for example, a severe radiation loss $d Q / d t$ would lead to a large compression (large negative value of $d r_{p} / d t$ ). In the extreme case, this leads to radiation collapse [2-6], with $r_{p}$ going rapidly to such small values that the plasma becomes opaque to the outgoing radiation, thus stopping further radiation loss.

This radiation collapse occurs at a critical current of 1.6 MA (the Pease-Braginski current) for deuterium [7,8]. For gases such as $\mathrm{Ne}$ or Ar, because of intense line radiation, the critical current is reduced to even below $100 \mathrm{kA}$, depending on the plasma temperature $[5,6,9,10]$. 
http://wjst.wu.ac.th

To show the effects of thermodynamics on the dynamics, the following numerical experiments are carried out for a case of neon operated with parameters of significant radiation:

The code is run with neon parameters with the value of $\gamma$ fixed at $\gamma=5 / 3$ (treating neon plasma as a perfect gas for the range of temperature during axial and radial phases) and effective charge $z_{\text {eff }}$ of 0 . The computed results are recorded. Under these conditions, radiation is negligible. (See Figure 6, trace 1; no thermodynamics and no radiation).

The code is run again with the same parameters as the earlier run, except that the values of $\gamma$ and $z_{\text {eff }}$ are calculated as they vary with temperature [11]. For this run, the $d Q / d t$ term is set to zero so that no radiation is emitted. (See Figure 6, trace 2; with thermodynamics but no radiation).

Finally the code is restored back to its standard form, with properly calculated values of $\gamma$ and radiation including radiation absorption. (See Figure 6, trace 3; with thermodynamics and radiation).

The computed results show the effects of thermodynamics and radiation (see traces $1,2,3$ ) on the pinch compression. For neon, effects of thermodynamics are subtle, effects of radiation are significant.

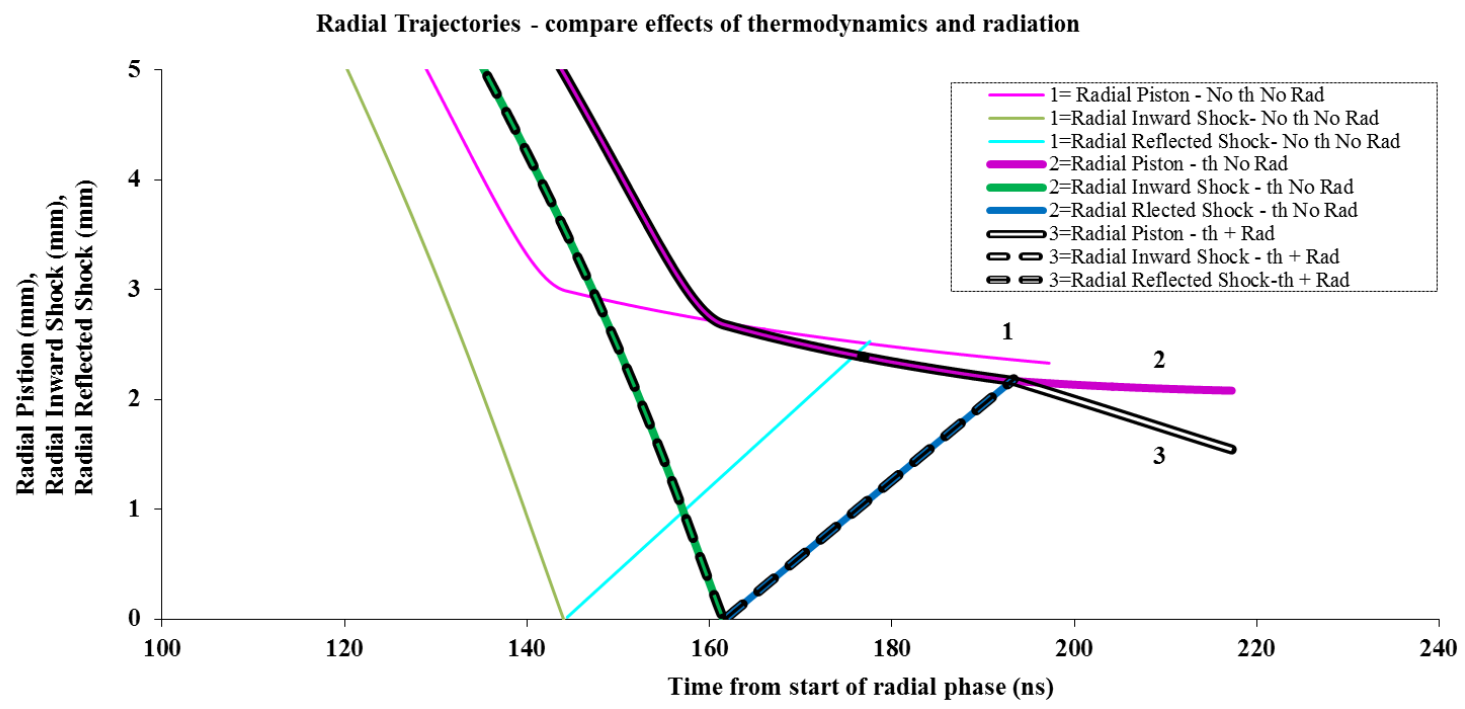

Figure 6 Comparison of the radial trajectories for hypothetical cases of:

(1) Trace 1 - no thermodynamics (th) and no radiation (Rad); (2) Trace 2 - with thermodynamics and no radiation; and (3) Trace 3 - with thermodynamics and radiation. 
http://wjst.wu.ac.th

\section{A dramatic example of radiatively enhanced compression in $\mathbf{~ K r}$}

A much more dramatic example of radiative collapse in $\mathrm{Kr}$ has been observed in a small $3 \mathrm{~kJ}$ DPF (INTI PF) on the basis of a current measurement is shown in Figure 7.

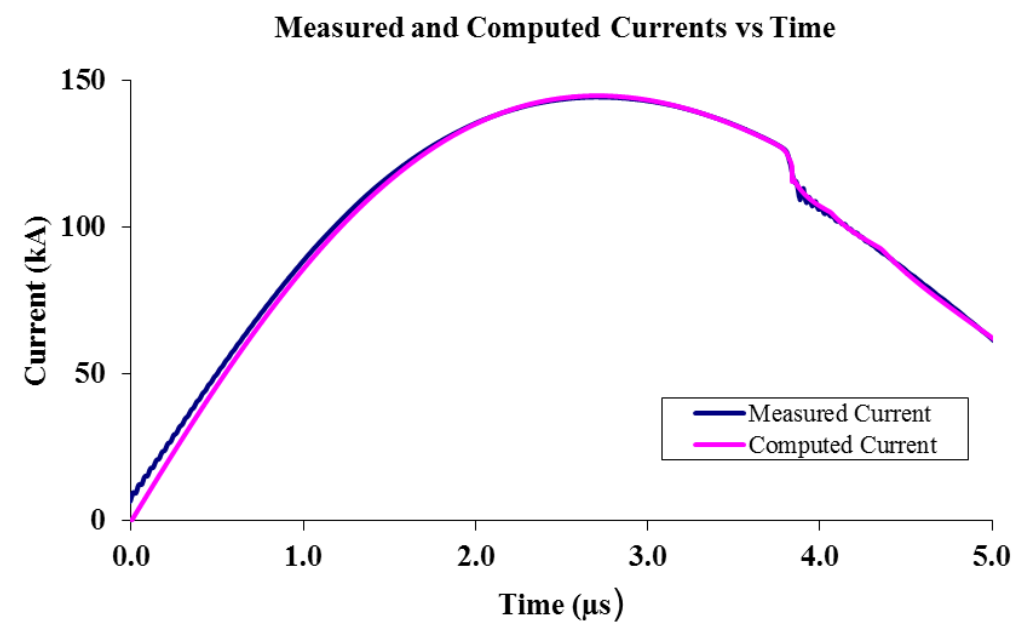

(a)

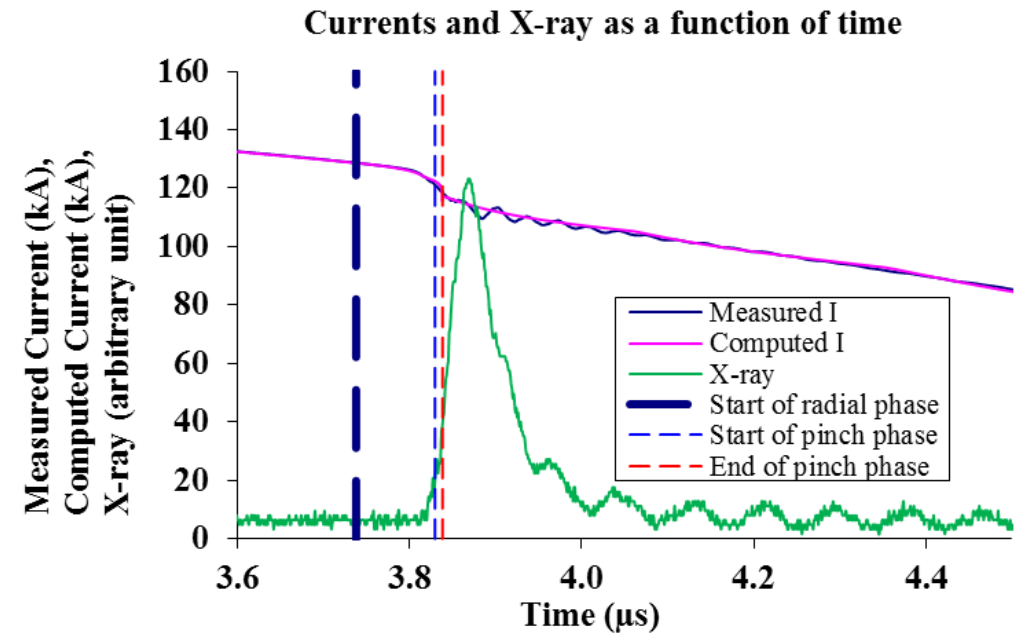

(b)

Figure 7 (a) Fitting the computed current trace to the measured current trace of INTI PF at $12 \mathrm{kV} 0.5$ Torr $\mathrm{Kr}$ (shot 631). (Note the 2 curves have a close fit. Without radiation, the current (not shown) has a much smaller dip.) and (b) Expanded view of the fitting with time markers and X-rays added in. 
http://wjst.wu.ac.th

Having fitted the computed current trace to the measured current trace, the resulting radial trajectory indicates strong radiative collapse to very small radius, as shown in Figure 8. The radial trajectory without radiation is also computed and shown for comparison.

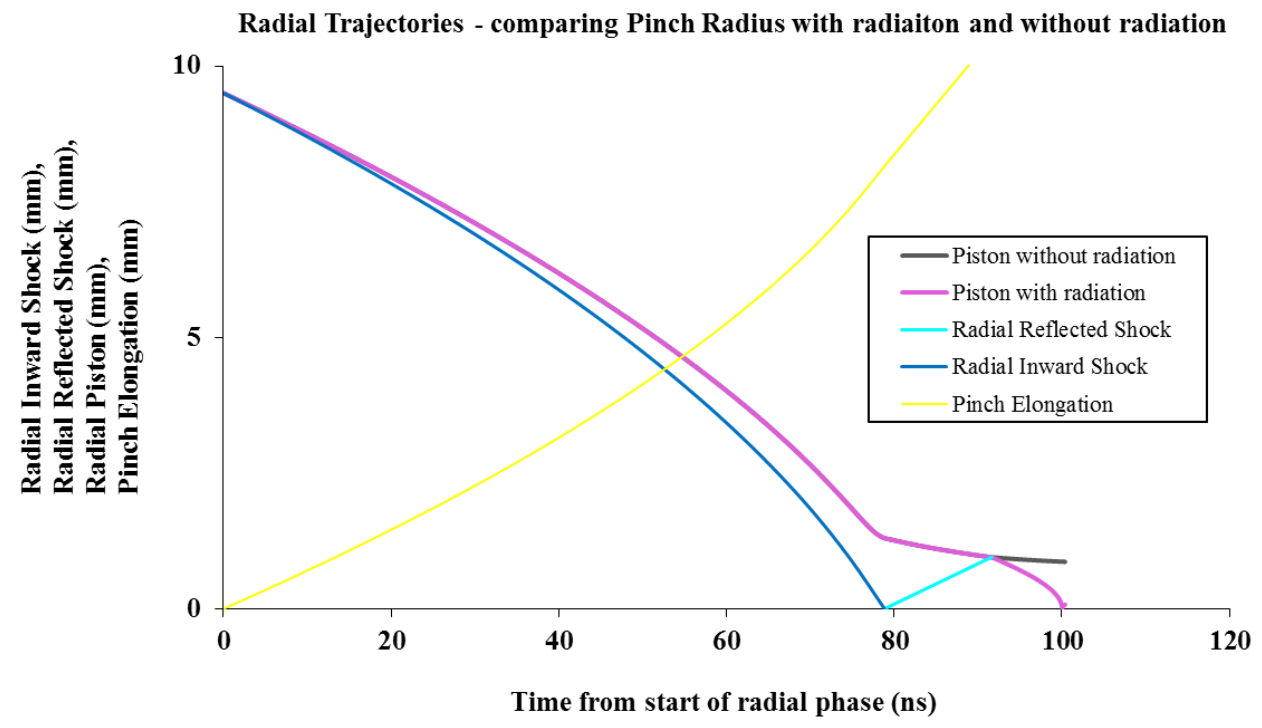

(a)

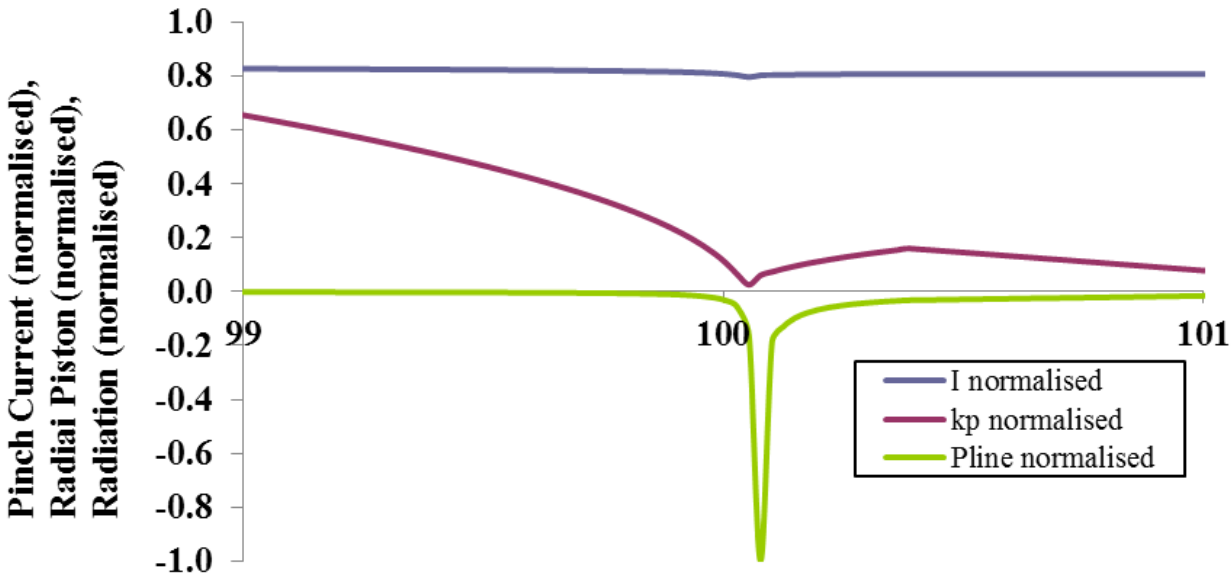

(b)

Figure 8 (a) Comparison pinch radial trajectory with hypothetical case of no radiation and (b) Normalised pinch radial trajectory vs pinch current profile and radiation profile [12] in ns time scale.

The peak compression region is magnified and shown in Figure 8a. The current values are normalized by $145 \mathrm{kA}$, the radiation Pline is normalized by $3.7 \times 10^{12} \mathrm{~W}$ and the radius ratio $k_{p}=r_{p} / a$ is multiplied by 20 . The pinch compresses to a radius of $0.0013 \mathrm{~cm}$, corresponding to a radius ratio (pinch radius normalized to anode radius) of 0.0014 . The radiative collapse is ended when plasma selfabsorption [12] attenuates the intense line radiation. The rebound of the pinch radius is also evident in 
http://wjst.wu.ac.th

Figure 8a. The line radiation leaving the plasma is also plotted (in normalized unit) to show its correlation to the trajectory in order to illustrate the effect of the radiation on the compression. This intense compression, despite the low mass swept in factor of $f_{m r}=0.11$, reaches $3.7 \times 10^{26}$ ions $\mathrm{m}^{-3}$, which is 15 times atmospheric density (starting from less than 1/1000 of an atmospheric pressure). Moreover the energy pumped into the pinch is $250 \mathrm{~J}$ whilst $41 \mathrm{~J}$ of radiation are emitted in several ns, most of the radiation occurring in a tremendous burst of 50 ps at peak compression with a peak radiation power of almost $4 \times 10^{12} \mathrm{~W}$. The energy density at peak compression is $4 \times 10^{13} \mathrm{~J} \mathrm{~m}^{-3}$ or $40 \mathrm{~kJ} \mathrm{~mm}^{-3}$. Thus even in-a small 3 kJ DPF intense High Energy Density (HED) is achieved with immense radiation power.

\section{Conclusion}

A capacitor bank generates a damped sinusoidal current when discharged into a short circuit. When powering a plasma focus, the top part of the current waveform is further damped and flattened by the axial motion of the current sheet. The radial phase, with severe rate of change of inductance due to a rapidly collapsing current sheet, is severely damped over a short period near the current peak. The waveform shows the signature current dip. Corresponding to the inductively-caused current dip is a sharp voltage spike which rises to a peak value greater than the charging voltage. This paper discusses the details of these effects. The subtle loading due to compressibility effects of non-perfect gas are also shown in a neon discharge, as are significant effects on the dynamics of radiation. Finally, from just a measured current waveform, the dramatic radiative collapse of the $K r$ pinch is demonstrated. The parameters of the HED state are deduced and presented, demonstrating a density compression of 15,000 times caused by a $4 \mathrm{TW}$ burst of radiation.

\section{References}

[1] SP Thong and S Lee. A Simplified method of switching a 2 mega-ampere capacitor bank using a voltage division technique. Malaysian J. Sci. 1973; 2, 157-69.

[2] S Lee. Plasma focus radiative model: Review of the Lee model code. J. Fusion Energ. 2014; 33, 319-35.

[3] S Lee. Radiative Dense Plasma Focus Computation Package: RADPF. Available at: http://www.plasmafocus.net/IPFS/modelpackage/File1RADPF.htm, accessed October 2017.

[4] S Lee and SH Saw. Course on plasma focus numerical experiments manual. In: Proceeding of the Joint ICTP-IAEA Workshop on Dense Magnetized Plasmas and Plasma Diagnostics. Trieste, Italy, 2010.

[5] S Lee, SH Saw and J Ali. Numerical experiments on radiative cooling and collapse in plasma focus operated in Krypton. J. Fusion Energ. 2012; 32, 42-9.

[6] M Akel and S Lee. Radiative collapse in plasma focus operated with heavy noble gases. J. Fusion Energ. 2013; 32, 111-6.

[7] R Pease. Equilibrium characteristics of a pinched gas discharge cooled by Bremsstrahlung radiation. Proc. Phys. Soc. 1957; 70, 11.

[8] SI Braginskii. The behavior of a completely ionized plasma in a strong magnetic field. Zh. Eksp. Teor. Fiz. 1957; 33, 645.

[9] K Koshelev and N Pereira. Plasma points and radiative collapse in vacuum sparks. J. Appl. Phys. 1991; 69, 21-44.

[10] S Lee, SH Saw, M Akel, HJ Kunze, P Kubes and M Paduch. Conditions for radiative cooling and collapse in plasma focus illustrated with numerical experiments on the PF1000. IEEE Trans. Plasma Sci. 2016; 44, 165-73.

[11] S Lee. Radius ratio of argon pinches. Aust. J. Phys. 1983; 3, 891-5.

[12] SH Saw and S Lee. Measurement of radiative collapse in $2.2 \mathrm{~kJ}$ PF: Achieving high energy density (HED) conditions in a small plasma focus. J. Fusion Energ. 2016; 35, 702-8. 